\title{
A randomised controlled trial to determine the effectiveness of double Tubigrip in grade 1 and 2 (mild to moderate) ankle sprains
}

\author{
B L Watts, B Armstrong
}

\begin{abstract}
Objective-To compare functional outcome in patients with acute grade 1 or 2 (mild to moderate) lateral ankle sprains randomised to treatment with or without a double tubigrip bandage (DTG).

Methods-400 patients presenting to the accident and emergency (A\&E) departments of a teaching hospital and a district general hospital and diagnosed with grade 1 or 2 lateral ankle sprains were randomised to treatment with or without a DTG bandage. A standardised telephone questionnaire was performed one week after presentation. The main outcome measures were: number of days until walking unaided, number of days off work, whether the injury kept the patient awake at night, whether analgesia was taken.

Results-197 of 400 patients completed follow up. There were no significant differences in terms of age, sex and occupation between the treatment groups. There were no significant differences between those who did and those who did not complete follow up. There was no significant difference between the treatment groups for number of days until walking unaided $(95 \% \mathrm{CI}-0.21$ to 0.88 days), number of days off work $(95 \%$ CI -0.70 to 1.02 days) or whether the injury kept the patient awake at night $(95 \%$ CI -10 to $17 \%)$. There was a significant difference between the groups in the use of analgesia $(95 \%$ CI 10 to $36 \%)$; the difference seemed to be that patients treated with DTG required significantly more analgesia.

Conclusions-Treatment of grade 1 and 2 ankle sprins with DTG does not seem to lead to a shorter time to functional recovery and may increase the requirement for analgesia.

(Emerg Med f 2001;18:46-50)
\end{abstract}

Emergency

Department, Queen

Alexandra Hospital,

Southwick Hill Road, Cosham, Portsmouth, Hampshire PO6 3LY, UK

B Watts

Emergency

Department,

Southampton General

Hospital,

Southampton

B Armstrong

Correspondence to:

Dr Watts, Specialist Registrar

(Beverley.Watts@,

ukgateway.net)

Accepted 6 October 2000
A grade 1 (mild) sprain comprises stretching of the ligament without macroscopic tearing and clinically this leads to little swelling or tenderness, with slight or no functional loss and no mechanical instability of the joint; recovery time is generally about three to seven days. ${ }^{3}$ Grade 2 (moderate) sprains are characterised by a partial macroscopic tear of the ligament, leading to moderate pain, swelling and tenderness. Some loss of motion and mild to moderate instability result, with recovery times of 10 to 21 days. A grade 3 (severe) sprain involves complete rupture of the ligament and this degree of injury consequently has a recovery time of six to eight weeks. Although this grading system for ankle sprains has been validated and found to be rather subjective, ${ }^{4}$ an improved and validated clinical alternative has yet to be put forward.

Once a bony injury has been excluded the usual treatment is to apply a compression bandage (typically double Tubigrip, DTG), give non-steroidal anti-inflammatory drugs if tolerated and advise the patient on rehabilitation exercises (the "RICE" regimen-rest, ice, compression and elevation). Although this approach has become the standard treatment over time, literature searching failed to identify any good evidence to support the use of DTG for grade 1 and 2 ankle sprains. Reducing postinjury oedema should control the amount of fibrin produced and thereby reduce scar tissue formation. ${ }^{5}$ However, the pressure exerted by a DTG is minimal over the sprained ligament ${ }^{6}$ and is insufficient to reduce or prevent oedema. ${ }^{78}$ One study ${ }^{9}$ found that elevating the limb at 45 degrees led to significant reduction in post-sprain oedema whereas elevation plus DTG or intermittent pneumatic compression both led to an increase in oedema. Unfortunately this paper suffers from having a small sample size. Another study ${ }^{10}$ suggested that patients who did not have a compression bandage had a shorter time to functional recovery than those treated with a compression bandage. There has been one study comparing the use of DTG with no treatment, ${ }^{11}$ which concluded that there was no difference in the course of pain, function, swelling or limitation of movement between the two groups. Unfortunately this study was not randomised.

All in all, there are no well conducted randomised controlled trials on the optimum treatment for this common injury. There are several studies looking at the treatment of ankle sprains in athletes ${ }^{212}$ or of using complex wraps, braces or intensive rehabilitation programmes. ${ }^{1314}$ However, the fact remains that such complicated treatment modalities lie outside the remit of most A\&E departments. DTG is the only support on offer and grade 1 or 2 ankle sprains have long been recognised to heal 


\section{Table 1 Exclusion criteria}

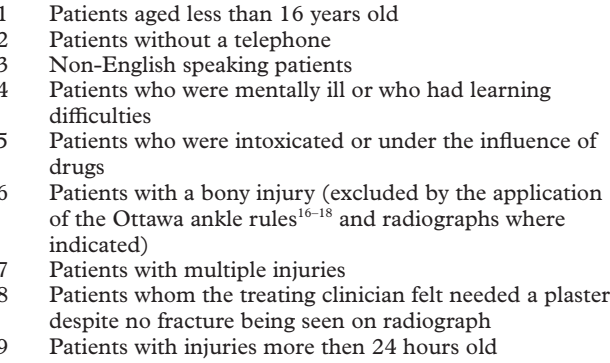

well with little or no rehabilitation and are therefore not routinely referred to our already heavily burdened physiotherapy departments. In addition, we know that a large number of patients who sustain a lateral ligament sprain of their ankle do not seek medical advice and that there is no evidence to suggest that such patients come to any harm.

Aside from the argument as to whether compression bandages are clinically indicated, there are other important factors that should be taken into consideration. It has been estimated that the annual cost of DTG alone for ankle sprains is $£ 654000$ in the UK. ${ }^{15}$ In addition to the cost in monetary terms, there is also the time cost for nursing staff measuring and applying the bandage and for patients waiting for their treatment to be given. The final overall cost for this non-evidence based "standard" treatment is therefore significantly greater.

The aim of this study was to look for a clinically significant $(10 \%)$ difference in terms of functional outcome between patients presenting with acute grade 1 and 2 ankle sprains treated with and without a double Tubigrip bandage.

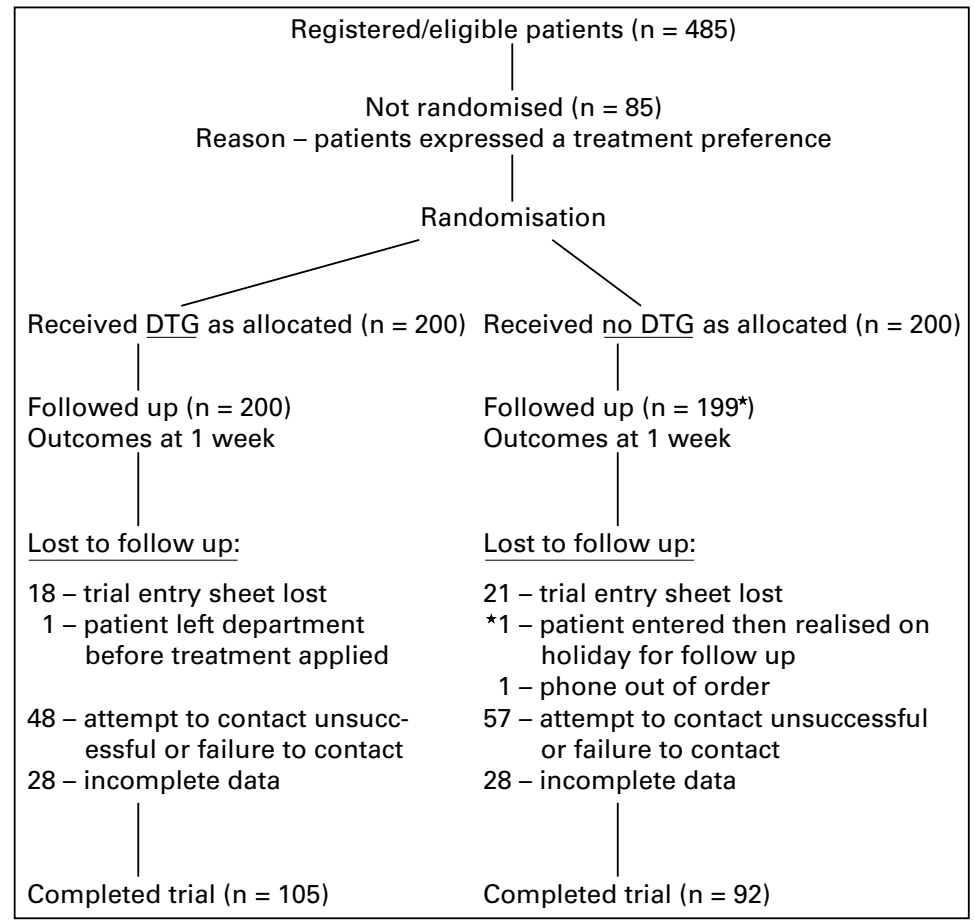

Figure 1 Consort diagram summarising patient flow through the trial.

\section{Methods}

SEARCH STRATEGY

The search terms "bandages, ankle sprain and compression" were used to search the Cochrane Database, Medline (1966 onwards for all databases), CINAHL and EMBASE databases. All relevant abstracts were reviewed and papers relevant or possibly relevant were retrieved and reviewed in full. In addition, the bibliographies of all retrieved papers were searched for relevant papers and the manufacturers of Tubigrip were contacted directly.

\section{STUDY DESIGN}

Patients presenting with ankle injuries to the A\&E department of the North Hampshire Hospital and the emergency department of Southampton General Hospital and given a final diagnosis of grade 1 or 2 lateral ankle sprain were eligible for entry into the study. Grade 3 sprains were rare (incidence not recorded separately) and were excluded from the trial. Table 1 shows the exclusion criteria used for grade 1 and 2 sprains.

After informed written consent was obtained a trial entry sheet was completed and the patient was randomised (see below) to receive either a DTG or no DTG. (Analgesia and rehabilitation advice were standardised between the two groups by means of an advice sheet which described exercises and advised simple analgesia if necessary). Patients were then telephoned by a member of the $A \& E$ reception staff one week after their attendance and a set of standardised questions was asked.

\section{OUTCOME MEASURES}

After reviewing the literature ${ }^{1011}{ }^{14}$ the following were chosen as the primary outcome measures:

1 Pain (sleep disturbance, need for analgesia)

2 Mobility (days until walking unaided, that is, no crutches, stick or holding onto furniture) 3 Need for and number of days off work

SAMPLE SIZE

This was based on the assumption (from previous studies ${ }^{2320}$ ) that grades 1 and 2 lateral ankle sprains take approximately 10 days to recover to a level where the patient can return to work. At a 5\% significance level with $80 \%$ power, predicting a $10 \%$ difference in this outcome measure (and time to return to "normal" everyday activities (excluding sport)) led to an estimated sample size of 200 patients in each group (treatment with and without a DTG).

The results were analysed using the independent $t$ test, $\chi^{2}$ or Fisher's exact test and the Mann-Whitney U test (for number of days off work) as appropriate. The SPSS ${ }^{19}$ and Arcus ${ }^{20}$ statistical packages were used for the statistical analyses.

\section{RANDOMISATION}

Randomisation was performed by a statistician from the Medical Statistics department of Southampton General Hospital using the SAS programme. Block randomisation was used. Four hundred sealed opaque brown envelopes with the trial number from 1 to 400 on the 
Table 2 Demographic details for patients who completed follow up

\begin{tabular}{|c|c|c|c|c|}
\hline & $D T G$ & No DTG & & $95 \% C I$ \\
\hline \multicolumn{5}{|l|}{ Age } \\
\hline Mean & 32.71 & 32.79 & NS, $p=0.96^{\star}$ & -3.29 to 3.14 years \\
\hline SD & 11.15 & 11.66 & & \\
\hline \multicolumn{5}{|l|}{ Sex } \\
\hline Male & 58 & 54 & $\mathrm{NS}, \mathrm{p}=0.57 \dagger$ & $-10 \%$ to $17 \%$ \\
\hline Female & 47 & 38 & & \\
\hline \multicolumn{5}{|l|}{ Occupation } \\
\hline Manual & 55 & 42 & NS, $p=0.32 \dagger$ & $-7 \%$ to $20 \%$ \\
\hline Sedentary & 44 & 44 & NS, $p=0.39 \dagger$ & $-20 \%$ to $8 \%$ \\
\hline Missing & 6 & 6 & NS, $p=0.39 \dagger$ & $-9 \%$ to $6 \%$ \\
\hline
\end{tabular}

${ }^{\star}$ Independent $t$ test, †Fisher's exact test.

front were used, with a single piece of white paper stating either "DTG or "No DTG" placed inside. The randomisation code was broken after recruitment into the trial had ceased.

\section{PATIENT ALLOCATION}

Patients were enrolled consecutively by $A \& E$ medical staff and emergency nurse practitioners (ENPs). The trial entry sheet was numbered from 1 to 400, with the correspondingly numbered brown envelope attached. Once the trial entry sheet was completed by the enrolling doctor/ENP, it was left for the treatment nurse to open the envelope and administer treatment as appropriate.

\section{Results}

It was noted that groups 1 and 9 of the exclusion criteria above were the most common reasons for exclusion and these accounted for approximately $20 \%$ of patients presenting with ankle injuries.

Figure 1 shows a Consort diagram ${ }^{21}$ to summarise participant flow through the trial.

There was no significant difference in terms of age, sex and occupation between patients treated with and without DTG who completed the trial (table 2). There was also no significant difference with respect to these variables between patients who completed and those who did not complete the trial (age: $t=0.75$ (95\% CI -2.12 to 4.69 years; sex: $p=0.67$, Fisher's exact test, $95 \% \mathrm{CI}-12 \%$ to $8 \%$; occupation: $p=0.96$, Fisher's exact test, 95\% CI $-1 \%$ to $20 \%$ ).

Table 2 shows the demographic details for patients who completed the trial.

Tables 3 and 4 show the results for the main outcome measures.

There was no significant difference between the treatment groups for whether pain from the injury kept the patient awake at night (table 3) but there was a significant difference in the numbers who took painkillers (table 3 ). The
Table 4

\begin{tabular}{|c|c|c|c|}
\hline & $D T G$ & \multicolumn{2}{|c|}{ No DTG } \\
\hline \multicolumn{4}{|c|}{ How many days off work? } \\
\hline Mean & 3.37 & 3.21 & NS, $p=0.94 \Omega$ \\
\hline SD & 2.33 & 2.02 & $\begin{array}{l}95 \% \mathrm{CI}-0.70 \text { to } 1.02 \\
\text { days }\end{array}$ \\
\hline \multicolumn{4}{|c|}{ How many days until walking unaided? } \\
\hline Mean & 2.65 & 2.32 & NS, $p=0.23^{\star}$ \\
\hline SD & 1.86 & 1.99 & $\begin{array}{l}95 \% \mathrm{CI}-0.21 \text { to } 0.88 \\
\text { days }\end{array}$ \\
\hline
\end{tabular}

^Independent $t$ test, $₫$ Mann-Whitney U test.

difference seemed to be that patients treated with DTG were more likely to take analgesia. There was no significant difference in the need for or number of days off work between the two treatment groups, or in the number of days until walking unaided.

\section{Discussion}

The management of sprained ankles with DTG has become a time honoured treatment for which good evidence from well conducted randomised controlled trials is lacking. Many studies fail to grade the severity of injuries they are studying, use inappropriate outcome measures or do not explain whether the assessor is blinded. ${ }^{22}$ Linde et $a l^{11}$ compared elastic compression bandage with no treatment in 100 patients and found no significant difference in the course of pain, function, swelling or limitation of movement between the two groups. However, this study has a major flaw as the patients were not randomised. Brooks et $a l^{10}$ randomised 102 patients to one of four treatment groups: no treatment, early physiotherapy, double Tubigrip and below knee plaster of Paris cast. Patients given no treatment returned to work earlier and had a lower "clinical score" than the other groups, but this study suffers from small numbers and no statistical analysis. The patients in the nonintervention groups may also have had less severe injuries and 15 of 27 patients in the no treatment group said they would have preferred some sort of support for the ankle. This latter point may be important and is a frequently quoted reason for giving DTG to patients with ankle sprains. It would be very useful in any future study to include patients who have expressed a treatment preference, in order to compare their outcomes with patients who were randomised to that treatment.

Our aim was to conduct a well designed randomised controlled trial to answer the question of whether treatment of grade 1 and 2 lateral ankle sprains with DTG leads to a more rapid return to functional recovery (as evidenced by the numbers of days taken to return to normal

Table 3

\begin{tabular}{|c|c|c|c|c|c|}
\hline & \multicolumn{2}{|l|}{$D T G$} & \multicolumn{2}{|l|}{ No DTG } & \\
\hline & Yes $(\%)$ & $N / A$ & Yes $(\%)$ & $N / A$ & \\
\hline Did the injury keep you awake at night? & $54(51.4)$ & & $44(47.8)$ & & $\begin{array}{l}\text { NS, } p=0.67 \dagger \\
95 \% \text { CI }-10 \% \text { to } 17 \%\end{array}$ \\
\hline Did you take pain killers? & $81(77.9)$ & & $50(54.3)$ & & $\begin{array}{l}\text { Significant, } p=0.001 \dagger \\
95 \% \text { CI } 10 \% \text { to } 36 \%\end{array}$ \\
\hline Did you need days off work? & $54(51.4)$ & $5(4.8)$ & $48(52.2)$ & $5(5.4)$ & $\begin{array}{l}\mathrm{NS}, \mathrm{p}=0.072 \ddagger \\
95 \% \mathrm{CI}-25 \% \text { to } 30 \%\end{array}$ \\
\hline
\end{tabular}

†Fisher's exact test, $¥ \chi^{2}$ test. 
activities, excluding sport) than no treatment. We wanted to look at the typical A\&E population (as opposed to athletes or military recruits) using "average" modalities for assessment and treatment (that is, not using stress radiographs or arthrograms). We chose grade 1 or 2 sprains because these are the commonest grade of injury encountered and because some clinicians prefer to immobilise grade 3 sprains in a below knee plaster of Paris for a short period of time. All junior doctors and ENPs were given teaching sessions on how to grade these injuries and were assessed to ensure consistency.

Our study had a follow up of 197 of 400 patients - that is, just under $50 \%$. For this reason we felt it was important to show that the groups who completed follow up were similar at the start of the trial (table 2) and similarly for those who did not complete follow up. The response rate was the same in the two treatment arms and is therefore unlikely to lead to an internally biased result, although we recognise that our power calculation required 400 subjects and our results therefore risk the introduction of a type II error. Although our follow up rate is comparable with other studies we feel that it could have been improved upon significantly by the use of a completely independent research assistant to perform the follow up telephone calls. A\&E reception staff (despite their best efforts) are notoriously busy and a significant number of patients were lost to follow up either because of a failure to contact or incomplete data collection once contacted. Clearly this will have had an impact on how generalisable our study results are.

Our study was set up to detect a $10 \%$ difference in outcome between the two treatment groups, as such a difference is one that most clinicians would accept as being significant. An equivalence study may actually be the ideal trial design to answer this clinical question, however the sample size required would be prohibitively large. We felt that as we did not find evidence of a significant difference in outcome between the two groups (except that the DTG group required significantly more analgesia) by using a sample size calculated to detect a $10 \%$ difference (see above for limitations) then this study design is acceptable.

Our results show that treatment of grade 1 and 2 ankle sprains without a DTG compression bandage does not result in a longer time to functional recovery or increased need for or numbers of days off work. It is interesting that the patients in our study required an average of only 3.37 versus 3.21 (DTG versus no DTG) days off work, which contrasts with the longer times quoted in other studies. ${ }^{2}{ }^{10}$ This should not reflect milder injuries, as the same grading system was used, but perhaps may be explained by a difference in the patient populations studied. The issues surrounding desire for a rapid return to work are complex and beyond the scope of this study.

The main conclusion from this study is that patients treated with DTG required significantly more analgesia for their sprains. This is perhaps surprising considering the claim that DTG provides analgesia by providing counterirritation to the skin. ${ }^{6}$ The increased need for analgesia may have been because the application of a DTG made these patients more aware of their injury or perhaps it reflects a real effect that such bandages may increase the discomfort if inadequate reapplication by the patient causes a tourniquet effect, for example. The finding that the use of DTG may actually be harmful is important and warrants further investigation.

As highlighted above, our conclusions must be viewed with some significant statistical limitations in mind. Our power calculation required 400 subjects but only half completed the trial and we used 10 days as the endpoint for return to work but the average number of days off work was only a third of this in our study. This means that our conclusions cannot be firm and perhaps this study should be considered as a pilot study, giving an indication of what might be expected if a larger study were to be undertaken. However, as pointed out above, we feel that an equivalence study may actually be the way to answer this research question, in which case the number of patients required is very likely to be prohibitively large.

\section{Conclusion}

This study suggests that the use of DTG compression bandage in grade 1 and 2 anskle sprains does not shorten recovery time or number of days off work. Application of DTG seems to be associated with an increase in the need for analgesia. This may be because the patient is more aware of their injury or it may be a real effect of the DTG, possibly because of inadequate reapplication by the patient.

The help of all A\&E staff and especially the receptionists of the North Hampshire and Southampton General Hospitals is gratefully acknowledged. Also many thanks to Miss Mo Collins, Jonathon Goddard and Bernie Higgins.

Funding: none.

Conflicts of interest: none.

Contributors

Dr Beverley Watts initiated the study hypothesis, discussed core ideas, designed the study protocol, participated in data collection and analysis and wrote the manuscript. Mr Bruce Armstrong initiated the study hypothesis, discussed core ideas, designed the study protocol and participated in data collection. $\mathrm{Mr}$ Michael Clancy discussed core ideas and interpretation of the data. The paper's guarantor is Mr Michael Clancy.

1 Trundle HR. Physiotherapy: the contribution. In: Skinner $\mathrm{D}$, Swain A, Peyton R, et al, eds. Cambridge textbook of accident and emergency medicine. Cambridge: Cambridge University Press, 1997:814.

2 Renstrom PAFH, Konradsen L. Ankle ligament injuries. $\mathrm{Br}$ F.Sports Med 1997;31:11-20.

3 Rourke K. The evaluation and treatment of acute ankle sprains. Fournal of Emergency Nursing 1994;20:528-35.

4 Lindenfield TN. The differentiation and treatment of ankle sprains. Orthopaedics 1988;11:203-6.

5 Hunter G. Specific soft tissue mobilisation in the treatment of soft tissue lesions. Physiotherapy 1994;80:15-21.

6 Tufft K, Leaman A. A better form of treatment? Professional Nurse 1994;11:745-6.

7 Sabri S, Roberts VC, Cotton LT. The effects of externally applied pressure on the haemodynamics of the lower limb. BMF 1971;3:503-8.

8 Thomas S. Bandages and bandaging-the science behind the art. CARE Science and Practice 1990;8:56-60.

9 Rucinski TJ, Hooker DN, Prentice WE, et al. The effects of intermittent compression on edema in postacute ankle sprains. F Orthop Sports Phys Ther 1991;14:65-9.

10 Brooks SC, Potter BT, Rainey JB. Treatment for partial tears of the lateral ligament of the ankle: a prospective trial. tears of the lateral ligan.

11 Linde F, Hvass I, Jurgensen U, et al. Compression bandage in the treatment of ankle sprains. Scand $\mathcal{F}$ Rehab Med 1984; 16:177-9. 
12 Mascaaro TB, Swanson LE. Rehabilitation of the foot and ankle. Orthop Clin North Am 1994;25:1447-60.

13 Wilkerson GB. Treatment of ankle sprains with external compression and early mobilisation. The Physician and Sports Medicine 1985;13:83-90.

14 Muwanga CL, Quinton DN, Sloan JP, et al. A new treatment of stable lateral ligament injuries of the ankle joint. Injury 1986;17:380-2.

15 Wilson S, Cooke M. Double bandaging of sprained ankles. BMF 1998;317:1722-3

16 Stiell I, Wells G, Laupacis A, et al. Multicentre trial to introduce the Ottawa ankle rules for use of radiography in acute ankle injuries. BMF 1995;311:594-7.
17 Stiell IG, McKnight D, Greenberg GH, et al. Implementation of the Ottawa ankle rules. $\mathcal{F A M A} 1994 ; 271: 827-32$.

18 Salt P, Clancy $M$. Implementation of the Ottawa ankle rules by nurses working in the accident and emergency department. F Accid Emerg Med 1997;14:363-5.

19 SPSS Inc. SPSS Version 8.0. Chicago: SPSS, 1998. Longman Software Publishing. Arcus Quickstat Biomedical
Version 1.2. Cambridge: Longman, 1997.

the CONSORT rest 2 Ogilvie-Harris DJ, Gilbart M. Treatment modalities for soft tissue injuries of the ankle: a critical review. Clin $\mathcal{F}$ Sports Med 1995;5:175-86. 